\title{
Music may reduce anxiety during invasive procedures in adolescents and adults
}

\author{
Abstracted from \\ Lai HI, Hwang MJ, Chen CJ, Chang KF, Peng TC, Chang Fm. \\ Randomised controlled trial of music on state anxiety and physiological indices in patients undergoing root canal treatment. \\ J Clin Nurs 2008; 17: 2654-2660 \\ Address for correspondence: Hui-ling Lai, Department of Nursing, Tzu Chi University, Hualien, Taiwan. \\ e-mail: snowjade@mail.tcu.edu.tw
}

\section{Question: Does listening to music reduce anxiety in patients undergoing root canal treatment?}

Design A block randomised controlled trial was conducted. Intervention Patients in the music (test) group listened to selected sedative music using headphones throughout the root canal treatment procedure. The control group subjects wore headphones but without the music.

Outcome measure Anxiety was measured before the study and at the end of the treatment procedure. Patients' heart rate, blood pressure and finger temperature were measured before the study and every 10 min until the end of the root canal treatment procedure.

Results The results revealed that there were no significant differences between the two groups for baseline data and procedure-related characteristics, except for gender. The subjects in the music group, however, showed a significant increase in finger temperature and a decrease in anxiety score over time compared with the control group. The effect size for state anxiety and finger temperature was 0.34 and 0.14 , respectively.

Conclusions Relaxing music administered through headphones to subjects during root canal treatment decreased the procedure-related anxiety of the patients and significantly increased finger temperature, but does not significantly affect blood pressure and heart rate over the procedure.

\section{Commentary}

This study is designed well, using valid trial methods, particularly making a concerted effort to measure the dependent variable in several ways to allow for triangulation of the effect (see www.phru.nhs.uk/ doc_links/rct\%20appraisal\%20tool.pdf for the critical appraisal skills programme article "making sense of evidence about clinical effectiveness: 11 questions to help you make sense of a trial"). The blinding of participants in trials involving psychological interventions is problematic, though. It is often apparent to the participant that they are receiving an active intervention. This study is a typical demonstration of such difficulties, especially when considered alongside the ethical duty to inform participants of the purpose of a research project. For this reason, it is important to identify the exact words used to explain the purpose of the study. This explanation will induce expectations and placebo effects that can be difficult to control for.

Notwithstanding the present study, the evidence suggesting an effect of music on anxiety levels during invasive medical and dental procedures is equivocal. a synthesis of the findings of such studies is hampered by the heterogeneity of methods, from study populations comprising adults or children, the level of anticipated anxiety of participants, the procedure(s) undertaken, the range of measures of anxiety adopted, the choice of a control (or comparison) group, to whether or not the music was preferred or not preferred by participants. ${ }^{1}$

Studies of young children (up to age 6 years) undergoing either dental or medical procedures (such as immunisation injections) have generally found little or no effect of music on anxiety levels, as manifest in disruptive behaviour or self-report. ${ }^{2-4}$ Adolescents and adults, though, have been found to respond to music with lower levels of reported anxiety and pain. There is some suggestion that such effects may be dependent on the participant anticipating that the music will have a positive effect. ${ }^{5}$

A second area for discussion is the putative mechanism of any effect. Several authors have explored the impact of listening to music on physiological parameters of stress, including blood pressure, heart rate and biochemical markers. Other authors have explored the psychological mechanism of any effect, eg, whether the effect results from heightened relaxation or from distraction. a third possibility is the mobilisation of positive cognitive coping efforts through the anticipated benefits of listening to music. It is possible that such expectations only become manifest at a certain age.

The use of music to reduce the experience of pain and anxiety is likely to be effective in adults and young people, especially where the anticipated effects are clearly outlined prior to the intervention. There is little impact of whether the patient chooses the music themselves or has the music chosen for them, ${ }^{6}$ provided that the music has 'soothing' qualities.

JT Newton

King's College London, London, UK

1. Standley JM. Music research in medical/ dental treatment: meta-analysis and clinical applications. J Music Ther 1986; 23: 56-122.

2. Aitken JC, Wilson S, Coury d, Moursi AM. The effect of music distraction on pain anxiety and behavior in pediatric dental patients. Pediatr Dent 2002; 24: 114-1148.

3. Noguchi LK. The effect of music versus nonmusic on behavioral signs of distress and self-report of pain in pediatric injection patients. J Music Ther 2006; 43: 16-38.

4. Arts SE, Abu-Saad HH, Champion GD, et al. Age-related response to lidocaineprilocaine (EMLA) emulsion and effect of music distraction on the pain of intravenous cannulation. Pediatrics 1994; 93: 797-801.

5. Anderson WD. The effectiveness of audio-nitrous oxide-oxygen psychosedation on dental behavior of a child. J Pedod 1980; 5: 3-21.

6. Walworth DD. the effect of preferred music genre selection versus preferred song selection on experimentally induced anxiety levels. J Music Ther 2003; 40: 2-14. 